\section{COVID-19: when, along with aging, sex matters}

\author{
Virginia Boccardi, Patrizia Mecocci \\ Institute of Gerontology and Geriatrics, \\ Department of Medicine and Surgery, \\ University of Perugia, Santa Maria della \\ Misericordia Hospital, Italy
}

\begin{abstract}
Older persons are more susceptible to infection due to the age-related immunologic changes and the state of constitutive lowgrade inflammation. The rate of complications from the novel severe acute respiratory syndrome-coronavirus 2 (SARS-CoV-2) and its related coronavirus disease 2019 (COVID-19), is significantly higher in the elderly, with men as the most affected. It is known that women, in general, are less susceptible to viral infections complications thanks to three main differences in sex chromosomes, innate immunity, and steroid hormones. COVID-19 epidemiology in Italy further support that older women, even if frailer, may experience lower mortality than men, which extends the 'male-female health-survival paradox' to acutely ill patient groups.
\end{abstract}

\section{COVID-19: when, along with aging, sex matters}

The outbreak of novel severe acute respiratory syndrome-coronavirus 2 (SARS$\mathrm{CoV}-2$ ) and its related coronavirus disease 2019 (COVID-19) quickly turned into a pandemic with Italy as one of the most affected countries. The overall fatality rate of persons with confirmed COVID-19 in the Italian population, based on data up to October 13 , was $10.1 \% \quad(36,233$ deaths $/ 358,499$ cases). This rate is higher than that observed in other countries. One possible motivation is in the Italian population peculiar demographic characteristics, which differ from other countries. In 2019 in Italy (60.4 million inhabitants), approximately $23 \%$ of the population was aged 65 years or older. About one-sixth of the whole population lived in the Northern region of Lombardy, where life expectancy at birth is 82 years for men and 86.4 years for women. ${ }^{1}$

Older persons are more susceptible to infection, due to the age-related immunologic changes and the state of constitutive low-grade inflammation, defined as inflammaging. These changes lead elders to a commonly decreased ability to fight infections, as well as to an increased prevalence of autoimmunity and diminished response to vaccines. The most affected subjects are the older ones with a median age of 61 years. The rate of complications from COVID-19 is significantly higher in older adults with a considerable gender difference, with men more affected. Statistics collected worldwide, in fact, lead to the evident conclusion that SARS-CoV-2 infection produces different effects in men and women. This aspect emerges both from the percentage of infections and from mortality. Specifically, in China the lethality rate of confirmed cases was $4.7 \%$ in men compared to $2.8 \%$ in women. ${ }^{2}$ The data available on October 13, published by the Italian National Health Institute (ISS), reported a consistently higher number of deaths in men in all age groups, but not after 90 years, when women outnumbered men (Figure 1A). However, considering the percentage of deaths on the total number of cases (men: 173,180; women: 185,300), the percentage of death in men was consistently higher and almost double in all age ranges as compared with women as shown in (Figure 1B).

Studies on the biology of viral infection and clinical management of the disease have demonstrated that differences in COVID-19 prevalence and severity of clinical expression is associated with sex. So, these data seem to indicate that there might be a sex predisposition to COVID-19 infection and complications, with men more prone to being affected at all ages.

It is known that women, in general, are less susceptible to viral infections thanks to three main differences as compared with men: i) sex chromosomes; ii) innate immunity; and iii) steroid hormones. ${ }^{3}$

The presence of two $\mathrm{X}$ chromosomes supports the immune system's strength even if one is inactive. ${ }^{4}$ In rodents and humans, females exhibit more efficient humoral and cell-mediated immune responses to antigenic stimulation, both vaccination, and infection, as compared with males. ${ }^{5}$

Clinical studies also revealed that males have lower CD3+ and CD4+ cell counts, CD4+/CD8+ cell ratios, and inflammatory helper T cell type 1 (Th1) responses. ${ }^{6}$ Thus, females exhibit higher cytotoxic $\mathrm{T}$ cell activity along with an upregulated expression of antiviral and pro-inflammatory genes, many of which have estrogen response elements in their promoters. ${ }^{7}$ Also, women generally produce higher levels of antibodies, which remain longer in the circulation. Studies of both humans and rodents have documented that the number
Correspondence: Virginia Boccardi, Institute of Gerontology and Geriatrics, Department of Medicine and Surgery, University of Perugia, Santa Maria della Misericordia Hospital, Piazzale Gambuli 1, 06132 Perugia, Italy. Tel.: +39.0755783524

E-mail: virginia.boccardi@unipg.it

Key words: Aging; older; COVID-19; sex; frailty; geriatrics.

Contributions: VB, conceived and wrote the manuscript; PM, reviewed the manuscript.

Conflicts of interest: the authors declare no potential conflicts of interest.

Received for publication: 23 October 2020. Accepted for publication: 25 November 2020.

This work is licensed under a Creative Commons Attribution-NonCommercial 4.0 International License (CC BY-NC 4.0).

${ }^{\circ}$ Copyright: the Author(s), 2020

Licensee PAGEPress, Italy

Geriatric Care 2020; 6:9421

doi:10.4081/gc.2020.9421

and activity of innate immune cells, including monocytes, macrophages, and dendritic cells (DCs) as well as inflammatory immune responses, in general, are higher in females as compared with males. ${ }^{8}$ Moreover, there are also differences between sexes in the induction of genes associated with toll-like receptor (TLR) pathways and antiviral type I interferon (IFN) responses. The intensity of activation of the immune cells is higher in women than in men, and it is correlated with the trigger of TLR7 and the production of IFN. TLR7 is expressed in innate immune cells, which recognize the single-strand RNA virus by promoting the production of antibodies and the generation of pro-inflammatory cytokines, including IL-6 and IL-1 family members. Indeed, in women, the amount of inflammatory IL-6 after a viral infection is lower than in males and is often correlated with longevity.

Sex hormones also affect the immune system via their effects on multiple immune cell subsets as well as on stromal cells. . $^{3,9,10}$ The positive effects of estrogen are wideranging and include maintaining muscle strength and promoting skeletal muscle repair, ${ }^{11}$ controlling glucose and lipid metabolism, ${ }^{12}$ and lowering the risk of cardiovascular diseases (CVDs). From these data, it is possible to notice an increase (although not significant) of the mortality rate in women that starts after the menopause, when the protective role of estrogens decline. The pres- 
ence of cardiovascular diseases, more frequent in men but also in post-menopausal women, worsens the prognosis after infection by SARS-CoV-2.

Another difference between sexes is in the expression of the angiotensin-converting enzyme 2 (ACE2) receptors. ACE2, the first known human homolog of angiotensinconverting enzyme (ACE), is a crucial modulator of the renin-angiotensin system (RAS) that regulates arterial pressure, electrolyte balance, and systemic vascular resistance. But ACE2 is also the entrance door for SARS-CoV-2 in lung and other organs, such as the bowel. SARS-CoV-2, via its spike protein, binds to the ACE2 allowing the virus to invade cells. In the lungs, the activation of the local RAS influences the pathogenesis of lung damage through multiple mechanisms, such as an increase in vascular permeability and alterations of alveolar epithelial cells. Activation of lung RAS involves renin, the initial enzyme of the RAS activation cascade. Renin splits the angiotensinogen by generating angiotensin I (Ang I, a decapeptide hormone, inactive). ACE converts Ang I to the active form angiotensin II (Ang II, an octapeptide hormone) which causes vasoactive effects by binding to the type I (AT1) and type II (AT2) receptors. Interestingly, ACE2 has been shown protective in several models of lung damage, including SARSCoV-1-mediated lung injury. ${ }^{13,14}$ In fact, ACE2 knockout mice exhibited a more severe disease compared with wild-type mice. ${ }^{15}$ The lack of ACE2 expression resulted in enhanced vascular permeability,

\section{A}

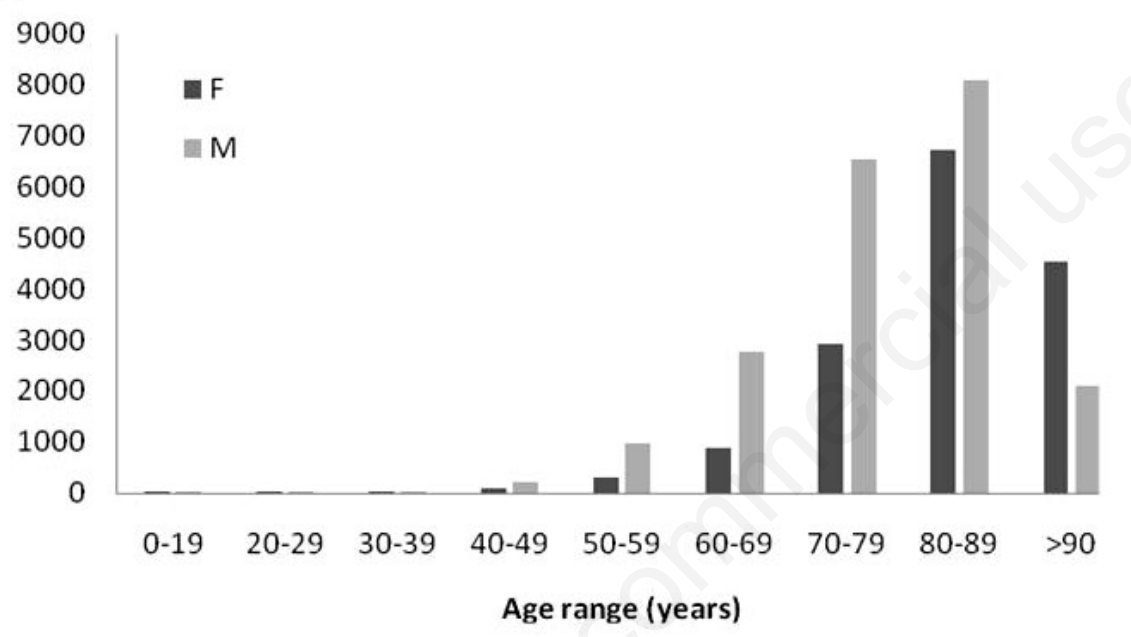

B

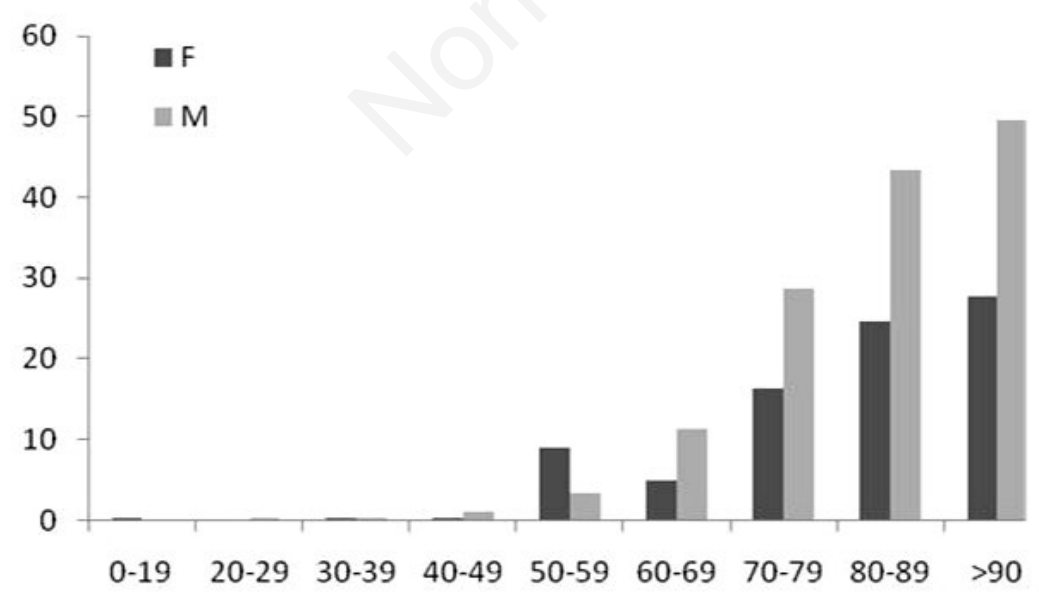

Age range (years)

Figure 1. A) Absolute number of deaths by COVID-19 infection; B) Lethality \% by COVID-19 infection stratified by age range and sex on available data until October 13, 2020 in the Italian population. M, male; F, female. Adapted from: https://www.epicentro.iss.it/coronavirus/sars-cov-2

increased edema, neutrophil accumulation, and worsened lung function. Mechanistically, the negative regulation of Ang II levels by ACE2 accounts, in part, for the protective role of ACE2 in ARDS. Thus during SARS-CoV-2 infection, it seems that the virus spike protein inhibits the expression of ACE2, abolishing its protective function. Since more elevated ACE2 receptors have been found in young and female rat lung, it is possible to hypothesize that young women, despite the higher door of entry, are less susceptible to SARS-CoV-2 complications during infection. ${ }^{16}$ Interestingly, the gene for ACE2 is located on the X chromosome, which raises the possibility that the presence of two X chromosomes could impact ACE2 activity, but this hypothesis needs further investigations.

From these data, in Italy the risk of dying from COVID-19 is constantly higher in men at every age. All these findings suggest that COVID-19 could be considered a sexually dimorphic condition, taking into account that prevalence, disease course, and outcome vary between men and women. Biological sex can impact individual vulnerability to immunemediated disease, including COVID-19. In general, women have a more robust immune response than men, which may help to better fight off infections. This could be a result of genetic and hormonal influences. However, it is interesting that the current data showing that women have better survival rates than men, also apply to subjects in the 80-plus age groups, when female sex hormone levels are low in women. ${ }^{17}$ These date further support previous literature demonstrating that women even in the hospital setting women were frailer, but experienced lower in-hospital and oneyear mortality than men suggesting important differences between the sexes and extending the male-female health-survival paradox to acutely ill patient groups. Precisely how biological sex makes a person resilient to diseases, such as COVID-19, remains to be elucidated. Future basic research and clinical trials in people need to consider biological sex as well as interactions with gender as an essential variable, with the ultimate aim of guaranteeing each person the best-personalized care.

\section{References}

1. Statista.it. Life expectancy at birth in Italy in 2019, by region. Available from: https://www.statista.com/statistics/5690 66/life-expectancy-at-birth-in-italy-byregion/ Accessed: 24 April 2020.

2. Epidemiology Working Group for NCIP Epidemic Response, Chinese 
Center for Disease Control and Prevention. [The epidemiological characteristics of an outbreak of 2019 novel coronavirus diseases (COVID-19) in China]. Zhonghua Liu Xing Bing Xue Za Zhi 2020;41:145-51.

3. Klein SL. Sex influences immune responses to viruses, and efficacy of prophylaxis and treatments for viral diseases. BioEssays 2012;34:1050-9.

4. Atzmon G, Cho M, Cawthon RM, et al. Evolution in health and medicine Sackler colloquium: Genetic variation in human telomerase is associated with telomere length in Ashkenazi centenarians. Proc Natl Acad Sci U S A 2010; 107:1710-7.

5. Klein SL, Jedlicka A, Pekosz A. The Xs and $\mathrm{Y}$ of immune responses to viral vaccines. Lancet Infect Dis 2010;10:338-49.

6. Regitz-Zagrosek V, ed. Sex and gender differences in pharmacology. BerlinHeidelberg: Springer-Verlag; 2012. Accessed: 24 April 2020.
7. Klein SL. Sex influences immune responses to viruses, and efficacy of prophylaxis and treatments for viral diseases. BioEssays 2012;34:1050-9.

8. Xia HJ, Zhang GH, Wang RR, Zheng YT. The influence of age and sex on the cell counts of peripheral blood leukocyte subpopulations in Chinese rhesus macaques. Cell Mol Immunol 2009;6: 433-40.

9. Olsen NJ, Gu X, Kovacs WJ. Bone marrow stromal cells mediate androgenic suppression of B lymphocyte development. J Clin Invest 2001;108:1697-704.

10. Klein SL, Flanagan KL. Sex differences in immune responses. Nature Rev Immunol 2016;16:626-38.

11. Horstman AM, Dillon EL, Urban RJ, et al. The role of androgens and estrogens on healthy aging and longevity. J Gerontol A Biol Sci Med Sci 2012;67: 1140-52.

12. Faulds MH, Zhao C, Dahlman-Wright K, Gustafsson J-Å. The diversity of sex steroid action: regulation of metabolism by estrogen signaling. J Endocrinol 2012;212:3-12.

13. Imai $\mathrm{Y}$, Kuba $\mathrm{K}$, Penninger JM. Angiotensin-converting enzyme 2 in acute respiratory distress syndrome. Cell Mol Life Sci 2007;64:2006-12

14. Kuba K, Imai Y, Rao S, et al. A crucial role of angiotensin converting enzyme 2 (ACE2) in SARS coronavirus-induced lung injury. Nat Med 2005;11:875-9.

15. Imai $Y$, Kuba $K$, Rao $S$, et al. Angiotensin-converting enzyme 2 protects from severe acute lung failure. Nature 2005;436:112-6.

16. Xudong $X$, Junzhu $C$, Xingxiang W, et al. Age- and gender-related difference of ACE2 expression in rat lung. Life Sci 2006;78:2166-71.

17. Veronese N, Siri G, Cella A, et al. Older women are frailer, but less often die then men: a prospective study of older hospitalized people. Maturitas. 2019; 128: 81-86. 